\title{
A Survey on Approximations of One-Dimensional Gaussian $Q$-Function
}

\author{
Vo Nguyen Quoc Bao ${ }^{1}$, Luu Pham Tuyen ${ }^{1}$, Huynh Huu Tue ${ }^{2}$
}

${ }^{1}$ School of Telecommunications, Posts and Telecommunications Institute of Technology, Ho Chi Minh City, Vietnam

${ }^{2}$ School of Electronics and Telecommunications, International University, Ho Chi Minh City, Vietnam

Correspondence: Vo Nguyen Quoc Bao, baovnq@ptithcm.edu.vn

Manuscript communication: received 11 February 2015, accepted 13 July 2015

\begin{abstract}
Predicting the digital communication system performance plays a very important role in the process of system design. This performance is usually quantified by symbol error probability or bit error probability. Computing these probabilities in presence of Additive White Gaussian Noise requires to work with integrals involving the Gaussian $Q-$ function, which cannot be expressed in closed-form in terms of elementary functions. As a result, approximating the Gaussian $Q$-function in closed-form expressions with high accuracy becomes a necessity. In this paper, we give an overview about the Gaussian $Q$-function approximations and via some illustrating examples, we discuss their accuracy, tractability as well as their computational complexity.
\end{abstract}

Keywords- Gaussian $Q$-function, Erf function, Erfc function, approximation, Nakagami- $m$, Log-normal.

\section{INTRODUCTION}

In communication systems, unknown factors, e.g., noise, are often modeled as Gaussian random variables by using the well-known Central limit theorem [1]. Hence, in most cases, when analyzing the system performance normally in terms of probability of errors, ones usually arrive at solving the semi-infinite integral involving the right-tail probability of standard normal distribution known as $Q(x)$, generally written as

$$
\int_{0}^{\infty}\left[\prod_{i=1}^{N} Q\left(A_{i} x\right)\right] f(x) d x
$$

where $A_{i}$ is a certain constant, $N$ is a positive integer, $Q(x)$ is the Gaussian $Q$-function, $f(x)$ is the probability density function (PDF) of signal-to-noise ratio (SNR) of fading channels that is generally considered as Nakagami- $m$ for small-scale fading cases. For example, the conditional symbol error rate (SER) of differential encoded QPSK (DE-QPSK) on the additive white Gaussian noise (AWGN) channel is given by [2, eq. (8.39)]

$$
\begin{aligned}
P_{S}(\gamma)= & 4 Q(\sqrt{\gamma})-8 Q^{2}(\sqrt{\gamma}) \\
& +8 Q^{3}(\sqrt{\gamma})-4 Q^{4}(\sqrt{\gamma}),
\end{aligned}
$$

with $\gamma$ being the instantaneous SNR per symbol. The average SER over a fading channel is obtained by averaging (2) over the PDF of SNR of corresponding fading, $f_{\gamma}(\gamma)$, expressed as

$$
\bar{P}_{s}=\int_{0}^{\infty} P_{S}(\gamma) f_{\gamma}(\gamma) d \gamma
$$

It is obvious that by putting (2) into (3), we will encounter the expression in (1). Solving (3) in terms of closed-form expressions plays an important role in studying the communication system performance. Closed-form expression of the system performance is also necessary for system optimization. Unfortunately, it is well-known that the $Q$-function cannot be expressed in closed-form in terms of elementary functions. The integral in (1) may be solved using either alternative presentation forms of Gaussian $Q$-function or $Q$-function approximations/bounds.

It is shown in [2-5] that by using the Craig Qfunction [6] combining with the moment generating function (MGF) function of SNR of the fading, known as the MGF-based approach, the error rate of most modulation schemes can be derived either in closedform expressions or in terms of single integrals with finite limits.

Besides the MGF-based approach, an alternative is the PDF-based approach shown in (3), which is useful in many cases. In general, the PDF-based approach requires to represent the $Q$-function in a closed-form expression so that the expression in (3) can be solved. Many works related to exactly solving (1) in some special cases can be found in, e.g., [7-10]. For example, the work in [9] solved (1), when $A_{i}=A$ for all $i$, arbitrary integer $N$ and $f(x)$ being the probability density function (PDF) of Rayleigh fading with maximal-ratio-combining (MRC) or over Nakagami-m with integer fading index $m$. However, the closedform result obtained appears in terms of the Lauricella hypergeometric function of $N$ variables, which seems slightly complicated and may not be mathematically tractable. The study in [10] represented the result of (1) with dissimilar $A_{i}$ over Nakagami- $m$ fading, but $N$ is limited up to 3 , i.e., $N \leq 3$. Hence, generally speaking, 
the need of approximating or bounding the $Q$-function is actually necessary and so far, this problem has been still a hot topic and got high attention [11-21].

This paper aims to give an overview about $Q$ function approximation with emphasis on its applications. We also discuss the advantages and disadvantages of these approximations and compare them in terms of accuracy and mathematical tractability. The accuracy is defined as the degree of closeness of approximations of $Q$-function to the exact function while the tractability involves the flexibility in algebraic manipulations, which depends on the form of approximation expressions.

The survey is organized as following. The next section presents an overview of $Q$ function approximation. The accuracy and the mathematical tractability of approximation are discussed respectively in Section 3 and Section 4. We also shed light on applications of $Q$-function approximations in Section 4. In Section 5, the computation complexity for all approximations are studied. Section 6 envisions the future and closes the paper.

\section{An Overview on $Q$-Function APPROXIMATION}

We start with the definition of the Gaussian $Q$-function $Q(x)$, which is the tail probability of the standard normal distribution as shown in Figure 1. Formally, the Gaussian $Q$-function is defined as [22] follows:

$$
Q(x)=\frac{1}{\sqrt{2 \pi}} \int_{x}^{\infty} e^{\frac{-t^{2}}{2}} d t,
$$

and can be expressed in terms of the error function $\operatorname{erf}(x)$ as

$$
Q(x)=\frac{1}{2}-\frac{1}{2} \operatorname{erf}\left(\frac{x}{\sqrt{2}}\right),
$$

and the complementary error function $\operatorname{erfc}(x)$ as $[1,2]$

$$
Q(x)=\frac{1}{2} \operatorname{erfc}\left(\frac{x}{\sqrt{2}}\right) \text {. }
$$

In order to avoid confusing with other kinds of $Q$-function, such as Marcum Q-function [23], Nuttall $Q$-function [24] or alternative representation of Gaussian $Q$-function as Craig $Q$-function [6], the term " $Q$ function" should be understood hereafter as "onedimensional Gaussian $Q$-function" for convenience unless explicitly stated otherwise. Without loss of generality, the argument of $Q$-function under consideration is in the rang of $[0, \infty)$. Another case of negative argument can be derived based on the relationship as follows:

$$
Q(x)=1-Q(-x) \text {. }
$$

Among many different $Q$-function approximation forms reported in the literature, we classify them into three classes including Mills'-ratio-based form (MRBF), sum of exponential function based form (SEFB), and polynominal based form (PLBF) based on the form of the existing $Q$-function approximations as shown in Figure 2.

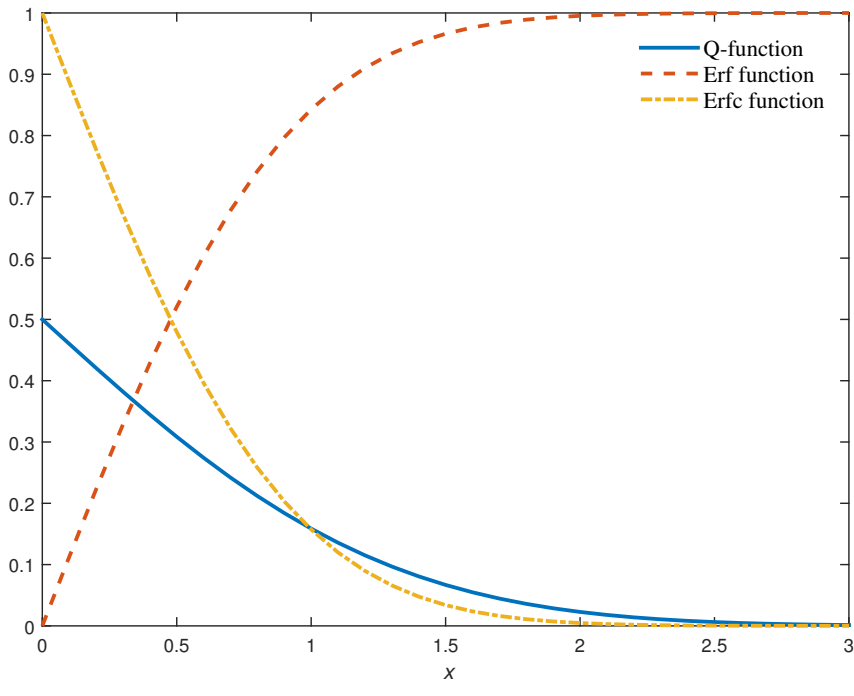

Figure 1. A plot of Q-function, $\operatorname{erf}(\cdot)$ function and $\operatorname{erfc}(\cdot)$ function.

\subsection{The Mills'-ratio-based Form}

We find out that there is a class of approximations explicitly related to the Mills' ratio [25-27]. We denote this class as the Mills'-ratio-based form (MRBF). The Mills' ratio, $r(x)$, is the ratio of the probability density function (PDF), $\varphi(x)$, to the reliability function, $\phi(x)$, of the standard normal law, where

$$
\varphi(x)=\frac{1}{\sqrt{2 \pi}} e^{\frac{-x^{2}}{2}},
$$

and

$$
\phi(x)=\frac{1}{2 \pi} \int_{x}^{\infty} \varphi(t) d t .
$$

From (8) and (9), we can rewrite the $Q$-function in terms of the Mills' ratio as

$$
Q(x)=\frac{r(x)}{\sqrt{2 \pi}} \exp \left(-\frac{x^{2}}{2}\right) .
$$

Equation (10) leads to form a class of $Q$-function (upper and lower) bounds by bounding the function $r(x)$. Also, approximating function $r(x)$ will result in approximating the $Q$-function. It should be noted here that the approximation results of the Mills' ratio $r(x)$ are commonly derived through empirical approaches.

We start with the approximations to $Q(\cdot)$ developed by Borjesson and Sundberg [11] as

$$
Q_{\mathrm{a}-\text { Borjesson }}(x)=\frac{1}{\sqrt{2 \pi}} \frac{1}{(1-a) x+a \sqrt{x^{2}+b}} e^{-\frac{x^{2}}{2}},
$$

where $a$ and $b$ are scalar fitting parameters.

Here, we denote $Q_{\mathrm{a}}(x)$ as the expression of the $Q$ function approximation. From (10) and (11), we can write $r(x)$ as follows:

$$
r(x)=\frac{1}{(1-a) x+a \sqrt{x^{2}+b}} .
$$

The parameters $a$ and $b$ in (12) can be determined by any numerical optimization procedure along with the 


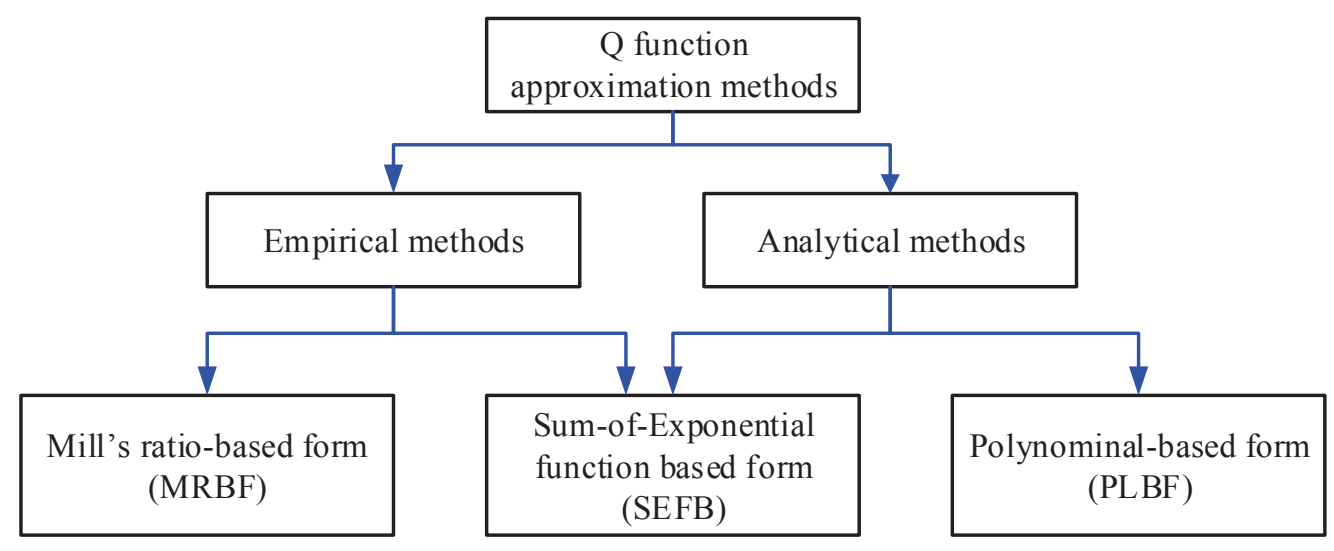

Figure 2. Classification of $Q$-function approximations.

constraint $Q(0)=0.5$. In particular, we can minimize the maximum absolute relative error (MARE), i.e.,

$$
\min _{a, b}|\mathcal{E}(x)|,
$$

where the absolute relative error $|\varepsilon(x)|$ is defined as

$$
|\varepsilon(x)|=\frac{\left|Q_{a}(x)-Q(x)\right|}{Q(x)} .
$$

Solving (13) is equivalent to find $a$ and $b$ for which $|\varepsilon(x)|$ is as small as possible for the considered interval of $x$. As shown in [11], the combination of $a=0.339$ and $b=5.510$, denoted as $Q_{\mathrm{a}-\text { Borjesson- } 1}(x)$, gives the best approximation for $x \geq 0$, namely

$$
Q_{\mathrm{a}-\text { Borjesson- } 1}(x)=\frac{1}{\sqrt{2 \pi}} \frac{1}{0.661 x+0.339 \sqrt{x^{2}+5.510}} e^{-\frac{x^{2}}{2}} \text {. }
$$

Another widely used approximation for $x \in[0, \infty)$ is $a=1$ and $b=1$ leading to

$$
Q_{\text {a-Borjesson-2 }}(x)=\frac{1}{\sqrt{2 \pi}} \frac{1}{\sqrt{x^{2}+1}} e^{-\frac{x^{2}}{2}} \text {. }
$$

$Q_{\mathrm{a}-\text { Borjesson-2 }}(x)$ in (16) is obtained by taking the geometric mean of the upper bound, i.e., [11, eq. (8)], and lower bound, i.e., [11, eq. (5)], of the $Q$-function. As stated in [11], the expression in (16) is quite useful as simple analytical approximation of $Q(x)$ for all $x \geq 0$. Other values of $a$ and $b$ for many different argument ranges can be found in [11, Table 1].

However, all approximations developed by Borjesson and Sundberg [11] are not suitable for algebraic manipulations related to communication system performance analysis. For example, it seems difficult for solving (1) with the PDF of Nakagami- $m$ fading. As pointed out in [11], this form is only applicable for programmable pocket calculators with the aim at improving the efficiency, e.g., minimizing the number of programing steps required.

Recently, an improvement in the sense of simplifying algebraic manipulations has been proposed in [12, 28], where the approximation form is given as

$$
Q_{\mathrm{a}-K a r a g i a n n i d i s}(x)=\frac{1}{\sqrt{2 \pi}} \frac{\left(1-e^{-A \frac{x}{\sqrt{2}}}\right)}{B x} e^{-\frac{x^{2}}{2}},
$$

In (17), $A$ and $B$ are derived numerically to minimize the integral of the absolute error given by

$$
\{A, B\}=\underset{\{A, B\}}{\arg \min } \frac{1}{R} \int_{0}^{R}\left|Q_{\mathrm{a}-\text { Karagiannidis }}(x)-Q(x)\right| d x,
$$

with $R$ being the range of argument values of interest. As stated in [12], $R=20$ is the value satisfying in most communication system applications, and the corresponding optimum values of $A$ and $B$ are 1.98 and 1.135 .

Taking the same form with [12, eq. (6)], a simple upper bound of the Gaussian has been very recently proposed by Jang in [19] as follows:

$$
Q_{\text {a-Jang }}(x)=\frac{1}{\sqrt{2 \pi}} \frac{\left(1-e^{-\sqrt{\frac{\pi}{2} x}}\right)}{x} e^{-\frac{x^{2}}{2}} \text {. }
$$

Comparing (19) to (17), we see that $A=\sqrt{\pi}$ and $B=1$. However, according to [19], expression (19) is an actual upper bound for $Q$-function while (17) is an approximation expression, i.e., neither upper bound nor lower bound. So, this is viewed as an advantage property of (19) over (17).

Nonetheless, the disadvantage of (17) as well as (19) is that the denominator contains the argument. As commented in [28], it is not quite convenient for integrating the expression over the PDF of Nakagami- $m$ fading. By this motivation, a slightly modified version of (17) was derived in [14]. By performing Taylor series expansion of the term $\exp (-A x / \sqrt{2})$ in (17), we have

$$
e^{-\frac{A x}{\sqrt{2}}}=\sum_{n=0}^{\infty} \frac{(-A x)^{n}}{\sqrt{2}^{n} n !}
$$

Substituting (20) into (17), the approximation expression can be expressed as [14] follows:

$Q_{\text {a-Isukapalli }}(x)=\frac{1}{\sqrt{2 \pi}}\left[\sum_{n=1}^{n_{a}} \frac{(-1)^{n+1}(A)^{n}}{B(\sqrt{2})^{n} n !} x^{n-1}\right] e^{-x^{2} / 2}$,

where $A$ and $B$ are selected according to [12]; $n_{a}$ is the number of selected terms depending on the desired tightness. 
It is straightforward to see that the form in (21) is more mathematically tractable than that of (17) in dealing with the Nakagami- $m$ fading, i.e., avoiding the presence of argument in the denominator and easily being integrable for any fading index $m$ of Nakagami$m$ fading. Furthermore, as stated in [14], by selecting appropriate parameter $n_{a}$, the tightness is comparable to that of (17).

\subsection{Sum-of-exponential-function-based form}

Together with the MRBF approximations, the approximations of $Q$-function can appear in sum of exponential functions form (SEFF). It was shown that the SEFF approximations are more flexible than the MRBF approximations.

The SEFF approximations are started with the Chiani approximation [13], which is based on the wellknown alternative representation of $Q$-function known as Craig $Q$-function [6] as follows:

$$
Q(x)=\frac{1}{\pi} \int_{0}^{\pi / 2} \exp \left(-\frac{x^{2}}{2 \sin ^{2} \phi}\right) d \phi .
$$

From (22), the complementary error function $\operatorname{erfc}(x)$ can be expressed as

$$
\operatorname{erfc}(x)=\frac{2}{\pi} \int_{0}^{\pi / 2} \exp \left(-\frac{x^{2}}{\sin ^{2}(\phi)}\right) d \phi .
$$

Using the monotonous increasing property of the function $\exp \left(-x^{2} / \sin ^{2} \phi\right)$ with $\phi \in[0, \pi / 2]$ and then making use the trapezoidal rule, $\operatorname{erfc}(x)$ with two immediate points of $\theta$ can be approximated as follows:

$$
\operatorname{erfc}(x) \simeq g(x, \phi)=\left(\frac{1}{2}-\frac{\phi}{\pi}\right) e^{-x^{2}}+\frac{1}{2} e^{-\left(x^{2} / \sin ^{2} \phi\right)} .
$$

The optimum value of $\phi$ is chosen to satisfy the following condition as

$$
\phi_{\mathrm{opt}}=\underset{\phi}{\arg \min } \frac{1}{R} \int_{0}^{R} \frac{|g(x, \phi)-\operatorname{erfc}(x)|}{\operatorname{erfc}(x)} d x,
$$

where $R$ is the considered range of argument values. With $R=20$, the value of $\phi_{\text {opt }}$ is $\pi / 3$. Hence, the approximation for $\operatorname{erfc}(x)$ is straightforwardly written as follows:

$$
\operatorname{erfc}(x) \simeq \frac{1}{6} e^{-x^{2}}+\frac{1}{2} e^{-4 x^{2} / 3} .
$$

From the relationship in (6), the approximation expression of $Q$-function in this case can be expressed as

$$
Q_{\text {a-Chiani }}(x)=\frac{1}{12} e^{-\frac{x^{2}}{2}}+\frac{1}{4} e^{-\frac{2 x^{2}}{3}} \text {. }
$$

Different from the approach used in [13], the work in [21] began with the formally $Q$-function definition in (4). By making a change of variable, i.e., $z=$ $(t-x) / \sqrt{2}$, the $Q$-function can be rewritten as follows:

$$
Q(x)=\frac{1}{\sqrt{\pi}} \int_{0}^{\infty} e^{-z^{2}} e^{-\sqrt{2} z} e^{-\frac{1}{2} x^{2}} d z .
$$

The semi-definite integral in (28) can be approximated by a using $N$-point Gauss-Hermite quadrature rule defined in [29]. It should be noted that the GaussHermite quadrature rule in [29] is defined in $[0, \infty)$, this is different from the conventional rule in [30] that defined in $(-\infty, \infty)$. Expression (28) can be rewritten as follows:

$$
Q_{\text {a-Shi }}(x)=\frac{1}{\sqrt{\pi}} e^{-\frac{1}{2} x^{2}} \sum_{n=1}^{N} \omega_{n} e^{-\sqrt{2} \zeta_{n} x},
$$

where $\zeta_{n}$ and $\omega_{n}$ are the $n$th root of $N$-order Hermite polynomial and the corresponding weights. As illustrated in [21], this approximation result is sufficiently accurate only with $N=2$, i.e., using only two Gauss points. Thus, in this case, the expression (29) reduces to two exponential terms form as in (27).

The works in [15, 17] provided a highly accurate result of (1) in sum of exponential by using Prony approximation, with $n=1$, Rayleigh and Rice fading with some common diversity combining techniques such as maximal ratio combining (MRC), equal-gain combining (EGC) and selection combining (SC). An approximation of $Q$-function also has been proposed in these works. In detail, they claim that the $Q$-function can be exactly represented by an infinite sum of exponential terms, expressed as follows:

$$
Q(x)=\lim _{N \rightarrow \infty} \frac{1}{N} \sum_{i=1}^{N} \frac{1}{2} e^{-a_{i} x^{2}},
$$

where

$$
a_{i}=\frac{1}{2} \sin ^{-2}\left(\frac{\pi(i-1)}{2(N-1)}\right) .
$$

According to (30), the Prony approximation of the Qfunction in terms of two exponential functions has represented in [17], namely

$$
Q_{\text {a-Loskot-1 }}(x)=0.208 e^{-0.971 x^{2}}+0.147 e^{-0.525 x^{2}} .
$$

A higher accurate result by adding one more exponential term is given by

$$
\begin{aligned}
Q_{\text {a-Loskot-2 }}(x)= & 0.168 e^{-0.876 x^{2}}+0.144 e^{-0.525 x^{2}} \\
& +0.002 e^{-0.603 x^{2}}
\end{aligned}
$$

An illustration in [17] shown that the result in (32) has comparable accuracy to the approximation in (30) with $N=50$.

In [18], a very simple result with only one exponential term was derived. However, different from all the above-mentioned SEFF approximations, which are based on analytical methods, it is from empirical method. In particular, originated from the principle of the result in (17) and (21), the approximation proposed in [18] is started with

$$
\frac{1-e^{-\frac{A}{\sqrt{2}} x}}{x} \simeq a e^{-b x},
$$

where $A$ was determined in [12] as 1.98 . 
In (34), the optimum values of fitting parameters $a$ and $b$ were given in [18] as 1.394 and 8/13 leading to

$$
Q_{\mathrm{a}-\text { Sofotasios }}(x)=0.49 e^{-\frac{8}{13} x} e^{-\frac{x^{2}}{2}} \text {. }
$$

It is straightforward to see that the result in (35) is simpler than that in (17) and further as concluded in [18], its accuracy is quite acceptable in general. Another similar result has recently proposed in [20], also based on the empirical approach, written as

$$
Q_{\mathrm{a}-\text { Benitez }}(x)=e^{a x^{2}+b x+c},
$$

where $a, b$ and $c \in R$ are fitting parameters derived by using numerical method.

Two criteria for finding the optimum fitting parameters are i) minimizing the sum of square errors (SSE) and ii) minimizing the MARE expressed in (14). Using MARE gives $a=-0.4920, b=-0.2887$, and $c=-1.1893$ for $x \in[0,20]$ while SSE criteria yields $a=-0.3842, b=-0.7640$, and $c=-0.6964$. Other optimum values of fitting parameter $(a, b, c)$ are provided in [20, Table 1].

Very recently, Dao et al. have proposed approximations for $Q(\cdot)$ function in the free and the exponential forms using genetic programming with semantic based crossover [31]. The proposed approximation is of the form

$$
Q(x)_{\mathrm{a}-\mathrm{Dao}}(x)=e^{a x^{6}+b x^{5}+c x^{4}+d x^{3}+e x^{2}+f x+g},
$$

where $a=-0.0000009, b=0000104, c=0.000686$, $d=0.015205, e=-0.363992, f=-0.762741$ and $g=-0.694072$. The numerical results in [31] shows that approximations in both forms are more accurate than all previous approximations designed by human experts.

\subsection{Polynomial-based form}

The work in [16] has shown that the MRBF and SEFF cannot be applied to log-normal channels despite their simplicity and tractable forms. The PDF of SNR on lognormal channel is given by [2]

$$
p(\gamma)=\frac{10 / \ln (10)}{\sqrt{w \pi \sigma^{2}} \gamma} \exp \left(-\frac{(10 \lg \gamma-\mu)^{2}}{2 \sigma^{2}}\right), \gamma>0,
$$

where $\mu$ and $\sigma$ are the logarithmic mean and logarithmic standard deviation of the shadowing, respectively.

It is obvious that the SEFF makes the exponential term in (38) becomes more complicated in derivation of the system performance. It is due to the need to make the integral of the product of (38) and the $Q$-function approximation over $[0, \infty)$ integrable, as shown in (1). By this motivation, the work in [16] has approximated the $Q$-function by a polynomial. In particular, the analysis in [16] is based on the observation that a sum of $n$ uniform random variables over $(-1 / 2,1 / 2)$ can be employed to approximate a Gaussian random variable with mean zero and variance $n / 12$ due to the central limit theorem [32]. The approximation expression is then given by

$$
\begin{aligned}
Q_{\mathrm{a}-C h e n}(x)= & 1-\sum_{m=0}^{n} \sum_{p=0}^{n} \frac{(-1)^{m+p}\left(\begin{array}{c}
n \\
p
\end{array}\right)}{m !(n-m) !}\left(\frac{n}{12}\right)^{p / 2} \\
& \times\left(\frac{n}{2}-m\right)^{n-p} x^{p} \\
& \times U\left[x-\sqrt{\frac{12}{n}}\left(\frac{n}{2}-m\right)\right],
\end{aligned}
$$

where $|x|<\sqrt{3 n}$ and $U(\cdot)$ is the unit step function defined as $U(x)=1$ if $x>0, U(x)=1 / 2$ if $x=0$ and $U(x)=0$ if $x<0$.

A closed-form result of derivation of symbol error rate over log-normal channel with $M$-PAM modulation scheme was given in [16]. It should be noted here that the MGF-based approach proposed in [2] also cannot derive the corresponding result in closed-form because the Laplace transformation of (38) cannot be obtained in closed-form. Thus, the approximation in (39) is rather useful in this scenario.

Before closing this section, we provide an summary of all $Q$ function approximations on Table I. An comparison among all $Q(\cdot)$ function approximations is also given on Table II and Figure 3.

\section{Accuracy Comparison}

The accuracy is one of the two important factors when approximating the $Q$-function. It is clearly shown that when using the empirical method, the fitting parameters strictly depend on the range of argument and hence, so does the accuracy. It is shown in [12] that the range of argument, which is appropriate for communication applications, is over $[0,20]$. In this section, we perform accuracy comparisons among approximations based on minimizing the MARE criteria given in (14). Unless otherwise specified, we set $n=2$ (the minimum number) to demonstrate its accuracy and efficiency.

The comparison of MRBF based $Q$-function approximations results are illustrated through Figure 4 and Figure 5. As shown in Figure 4, the Borjesson-1 expression in (15) is the best approximation over a wide range of argument. The accuracy of Karagianidis is comparable to that of Borjesson-1 only over the range of $[0,2]$ and better than Borjesson-2 over the range of $[0,3]$. However, as discussed, the form of Karagianidis is more mathematically tractable than that of Borjesson-1 and Borjesson-2. This is viewed as the cost of more tractable form of Karagianidis. Moreover, the approximation of Jang is better than Karagianidis with the argument of $x>3$ and is comparable to Borjesson-2. The advantage of Jang approximation is that it is an upper bound of the $Q$-function and when $x \rightarrow \infty$, the Jang's approximation approaches to zero while the one provided by Karagianidis approaches to a constant [19].

Figure 5 illustrates the accuracy comparison between Isukapalli's approximation to Karagianidis' one. It is clear that the accuracy of Isukapalli increases as increasing the number of terms $n_{a}$ in Taylor series, i.e., (21). 
Table I

A Summary on $Q(\cdot)$ Approximations

\begin{tabular}{|c|c|c|}
\hline \multirow{11}{*}{ 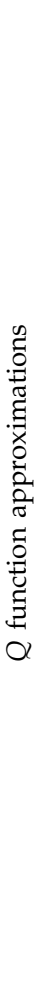 } & \multirow{4}{*}{ 竞 } & $Q_{\mathrm{a}-\text { Borjessson }}(x)=\frac{1}{\sqrt{2 \pi}} \frac{1}{(1-a) x+a \sqrt{x^{2}+b}} e^{-\frac{x^{2}}{2}}$ \\
\hline & & $Q_{\mathrm{a}-K a r a g i a n n i d i s}(x)=\frac{1}{\sqrt{2 \pi}} \frac{(1-e \sqrt{x})}{B x} e^{-\frac{x^{2}}{2}}$ \\
\hline & & $Q_{\text {a-Jang }}(x)=\frac{1}{\sqrt{2 \pi}} \frac{\left(1-e^{-\sqrt{\frac{\pi}{2}} x}\right)}{x} e^{-\frac{x^{2}}{2}}$ \\
\hline & & $Q_{\text {a-Isukapilli }}(x)=\frac{1}{\sqrt{2 \pi}}\left[\sum_{n=1}^{n_{a}} \frac{(-1)^{n+1}(A)^{n}}{B(\sqrt{2})^{n} n !} x^{n-1}\right] e^{-x^{2} / 2}$ \\
\hline & \multirow{6}{*}{ 壹 } & $Q_{\mathrm{a}-\text { Chiani }}(x)=\frac{1}{12} e^{-\frac{x^{2}}{2}}+\frac{1}{4} e^{-\frac{2 x^{2}}{3}}$ \\
\hline & & $Q_{\mathrm{a}-\mathrm{Shi}}(x)=\frac{1}{\sqrt{\pi}} e^{-\frac{1}{2} x^{2}} \sum_{n=1}^{N} \omega_{n} e^{-\sqrt{2} \zeta_{n} x}$ \\
\hline & & $Q_{\mathrm{a}-\text { Loskot }}(x)=0.208 e^{-0.971 x^{2}}+0.147 e^{-0.525 x^{2}}$ \\
\hline & & $Q_{\mathrm{a}-\text { Sofotasios }}(x)=0.49 e^{-\frac{8}{13} x} e^{-\frac{x^{2}}{2}}$ \\
\hline & & $Q_{\mathrm{a}-\operatorname{Benitez}}(x)=e^{a x^{2}+b x+c}$ \\
\hline & & $Q_{\mathrm{a}-\mathrm{Dao}}(x)=e^{a x^{6}+b x^{5}+c x^{4}+d x^{3}+e x^{2}+f x+g}$ \\
\hline & 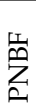 & $Q_{\mathrm{a}-\mathrm{Chen}}(x)=-\sum_{m=0}^{n} \sum_{p=0}^{n} \frac{(-1)^{m+p}\left(\begin{array}{c}n \\
p\end{array}\right)}{m !(n-m) !}\left(\frac{n}{12}\right)^{p / 2}\left(\frac{n}{2}-m\right)^{n-p} x^{p} U\left[x-\sqrt{\frac{12}{n}}\left(\frac{n}{2}-m\right)\right.$ \\
\hline
\end{tabular}

Table II

Comparison among All $Q(\cdot)$ Function Approximations

\begin{tabular}{|l|c|c|c|c|c|c|c|c|c|}
\hline & $x=0.1$ & $x=0.3$ & $x=0.5$ & $x=0.7$ & $x=1$ & $x=5$ & $x=10$ & $x=15$ & $x=20$ \\
\hline$Q(x)$ & $4.6017 \mathrm{e}-01$ & $3.8209 \mathrm{e}-01$ & $3.0854 \mathrm{e}-01$ & $2.4196 \mathrm{e}-01$ & $1.5866 \mathrm{e}-01$ & $2.8665 \mathrm{e}-07$ & $7.6199 \mathrm{e}-24$ & $3.6710 \mathrm{e}-51$ & $2.7536 \mathrm{e}-89$ \\
\hline$Q_{\mathrm{a}-\text {-Borjessson }}(x)$ & $3.9498 \mathrm{e}-01$ & $3.6530 \mathrm{e}-01$ & $3.1490 \mathrm{e}-01$ & $2.5581 \mathrm{e}-01$ & $1.7110 \mathrm{e}-01$ & $2.9157 \mathrm{e}-07$ & $7.6564 \mathrm{e}-24$ & $3.6790 \mathrm{e}-51$ & $2.7570 \mathrm{e}-89$ \\
\hline$Q_{\mathrm{a}-\text { Karagiannidis }}(x)$ & $4.5693 \mathrm{e}-01$ & $3.8415 \mathrm{e}-01$ & $3.1232 \mathrm{e}-01$ & $2.4552 \mathrm{e}-01$ & $1.6062 \mathrm{e}-01$ & $2.6174 \mathrm{e}-07$ & $6.7794 \mathrm{e}-24$ & $3.2486 \mathrm{e}-51$ & $2.4321 \mathrm{e}-89$ \\
\hline$Q_{\mathrm{a}-\text { Jang }}(x)$ & $4.6759 \mathrm{e}-01$ & $3.9842 \mathrm{e}-01$ & $3.2786 \mathrm{e}-01$ & $2.6056 \mathrm{e}-01$ & $1.7287 \mathrm{e}-01$ & $2.9678 \mathrm{e}-07$ & $7.6946 \mathrm{e}-24$ & $3.6871 \mathrm{e}-51$ & $2.7605 \mathrm{e}-89$ \\
\hline$Q_{\text {a-Isukapilli }}(x)$ & $4.5693 \mathrm{e}-01$ & $3.8416 \mathrm{e}-01$ & $3.1241 \mathrm{e}-01$ & $2.4595 \mathrm{e}-01$ & $1.6247 \mathrm{e}-01$ & $2.0881 \mathrm{e}-05$ & $2.2070 \mathrm{e}-20$ & $8.8628 \mathrm{e}-47$ & $2.9462 \mathrm{e}-84$ \\
\hline$Q_{\text {a-Chiani }}(x)$ & $3.3126 \mathrm{e}-01$ & $3.1511 \mathrm{e}-01$ & $2.8516 \mathrm{e}-01$ & $2.4556 \mathrm{e}-01$ & $1.7890 \mathrm{e}-01$ & $3.2500 \mathrm{e}-07$ & $1.6073 \mathrm{e}-23$ & $1.1553 \mathrm{e}-50$ & $1.1532 \mathrm{e}-88$ \\
\hline$Q_{\text {a-Shi }}(x)$ & $4.6017 \mathrm{e}-01$ & $3.8206 \mathrm{e}-01$ & $3.0838 \mathrm{e}-01$ & $2.4154 \mathrm{e}-01$ & $1.5767 \mathrm{e}-01$ & $1.6129 \mathrm{e}-07$ & $9.9883 \mathrm{e}-25$ & $8.5944 \mathrm{e}-53$ & $1.0270 \mathrm{e}-91$ \\
\hline$Q_{\text {a-Loskot }}(x)$ & $3.5222 \mathrm{e}-01$ & $3.3081 \mathrm{e}-01$ & $2.9209 \mathrm{e}-01$ & $2.4291 \mathrm{e}-01$ & $1.6573 \mathrm{e}-01$ & $2.9323 \mathrm{e}-07$ & $2.3273 \mathrm{e}-24$ & $7.3499 \mathrm{e}-53$ & $9.2358 \mathrm{e}-93$ \\
\hline$Q_{\mathrm{a}-\text {-Sofotasios }}(x)$ & $4.5846 \mathrm{e}-01$ & $3.8947 \mathrm{e}-01$ & $3.1789 \mathrm{e}-01$ & $2.4929 \mathrm{e}-01$ & $1.6062 \mathrm{e}-01$ & $8.4183 \mathrm{e}-08$ & $2.0086 \mathrm{e}-25$ & $6.6557 \mathrm{e}-54$ & $3.0629 \mathrm{e}-93$ \\
\hline$Q_{\text {a-Benitez }}(x)$ & $4.5995 \mathrm{e}-01$ & $3.8282 \mathrm{e}-01$ & $3.0899 \mathrm{e}-01$ & $2.4185 \mathrm{e}-01$ & $1.5809 \mathrm{e}-01$ & $7.3647 \mathrm{e}-07$ & $4.9426 \mathrm{e}-21$ & $1.5065 \mathrm{e}-43$ & $2.0853 \mathrm{e}-74$ \\
\hline$Q_{\mathrm{a}-\text {-Dao }}(x)$ & $4.6002 \mathrm{e}-01$ & $3.8210 \mathrm{e}-01$ & $3.0858 \mathrm{e}-01$ & $2.4200 \mathrm{e}-01$ & $1.5866 \mathrm{e}-01$ & $2.8672 \mathrm{e}-07$ & $9.0226 \mathrm{e}-24$ & $2.0201 \mathrm{e}-46$ & $5.4641 \mathrm{e}-36$ \\
\hline$Q_{\mathrm{a}-\text {-Chen }}(x)$ & $4.6139 \mathrm{e}-01$ & $3.8551 \mathrm{e}-01$ & $3.1352 \mathrm{e}-01$ & $2.4764 \mathrm{e}-01$ & $1.6381 \mathrm{e}-01$ & $1.8652 \mathrm{e}-14$ & $5.6843 \mathrm{e}-14$ & $1.3642 \mathrm{e}-12$ & $3.1832 \mathrm{e}-12$ \\
\hline
\end{tabular}

Recalling that the expression of Isukapalli is an improvement version of Karagianidis in terms of mathematical tractability by using Taylor series expansion with the purpose of canceling the argument in the denominator. It can be observed that there is an interval of arguments so that the Isukapalli's approximation coincide with that of Karagianidis. For example, the agreement interval for $n_{a}=20$ is about $[0,5]$ as shown in Figure 5.

The comparisons of the SEFB approximations are illustrated in Figure 6 and Figure 7. We see from Figure 6 that the Chiani's approximation with two exponential terms is the worst approximations as compared to Shi's and Loskot's. The approximation of Loskot, even with two terms, is better than that of Chiani. In Figure 6, we also plot the Shi's expression with $N$ increasing from 2 to 5. It is obvious that the accuracy of Shi's expression improves as increasing N. Furthermore, the Shi's approximation with only two terms, i.e., $N=2$, has comparable accuracy to the approximations provided by Chiani as well as Loskot. Since the exponential terms is limited to two, the accuracy of Shi's approximation is 


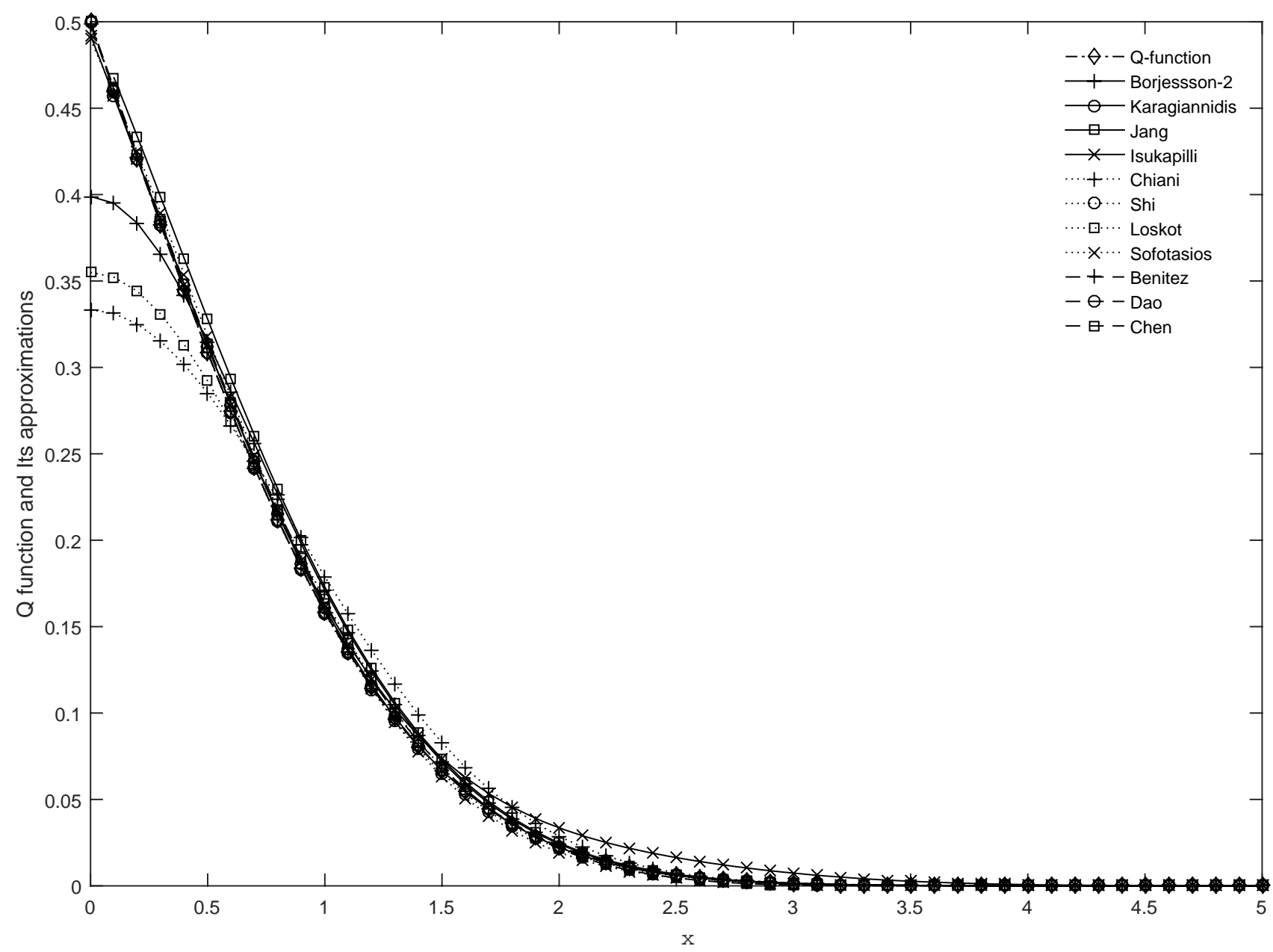

Figure 3. Comparison among all $Q(\cdot)$ function approximations.

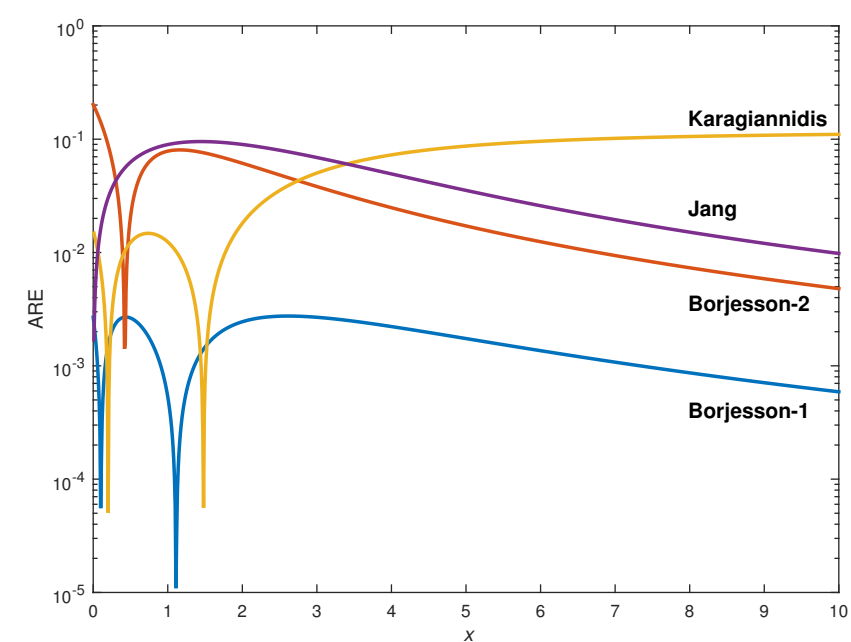

Figure 4. Comparison among Bojesson-1, Borjesson-2, Karagianidis and Jang approximations.

better than that of Loskot with $x \in[0,3]$ but is worsen with $x>3$. Moreover, with $x \geq 5$, these three approximation expressions are comparable to each other in terms of the accuracy as well as the tractability.

Figure 7 illustrates the accuracy comparisons of among Chiani, Sofotasios, Benitez-MARE and BenitezSSE approximations. It is shown that with $x<3$, the

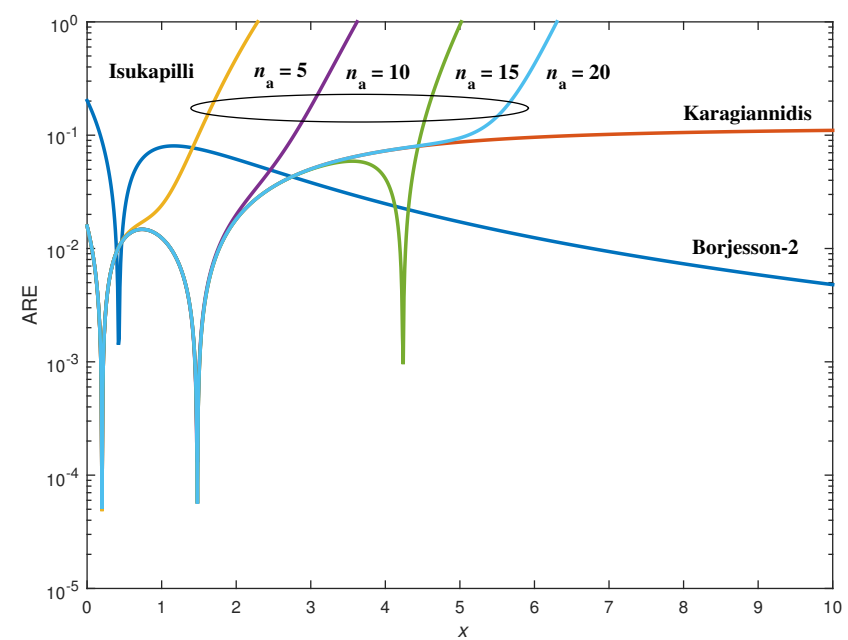

Figure 5. Comparison among Borjesson-2, Karagianidis and Isukapalli approximations.

approximation of Benitez according to SSE criteria is the best. But for wide range of argument, it can be observed that the Benitez's approximation according to MARE criteria is better. The Safotasios' approximation has same form as Benitez's one but it gives the worse accuracy compared to Benitez. Although the expression of Chiani contains two exponential terms but it is less 


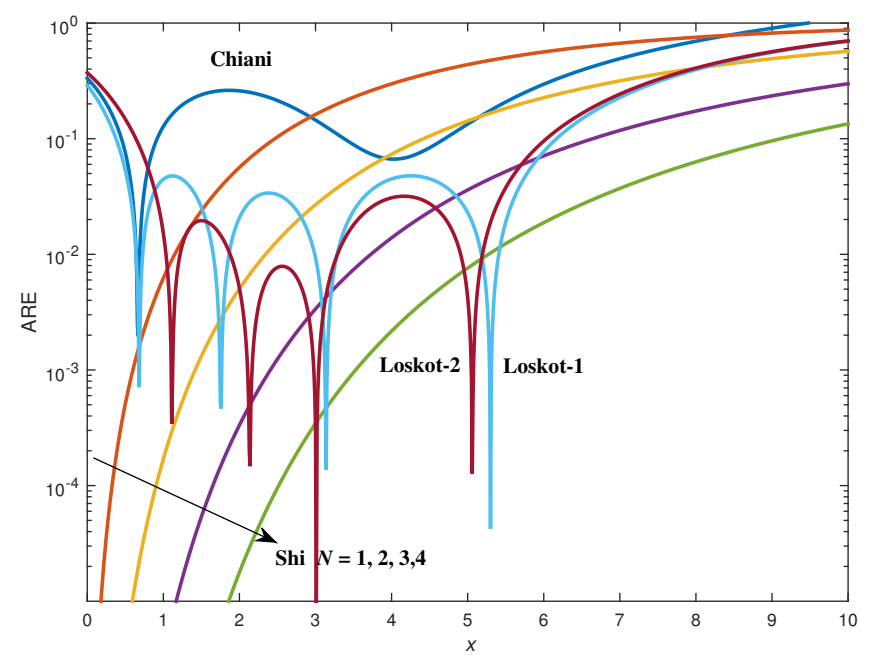

Figure 6. Comparison among Chiani, Shi and Loskot approximations.

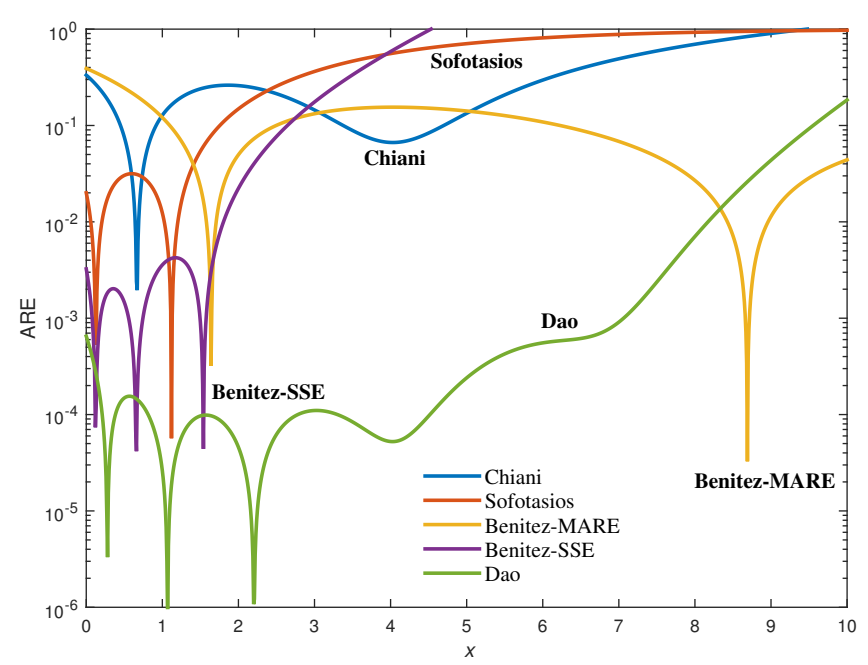

Figure 7. Comparison among Chiani, Loskot-1, Benitez-MARE and Benitez-SSE approximations.

accurate than the Benitez-MARE with only one term and only better than Benitez-SSE with $x>3$.

In Figure 8 , we plot the polynomial expression of Chen with 8 and 12 terms. Chiani and Benitez approximations are also included for references. We can see that the Chen's approximations provide good accuracy with small argument, i.e., $x \in[0,3]$. In addition, increasing $N$ does not give much improvement in accuracy. It is worth to note here that the accuracy for Chen's approximation associated with the range of argument directly depends on the number of terms $n$, that is, $|x|<\sqrt{3 n}$ as mentioned previously. For example, with $n=12$, the accuracy only can be observed on the range $x<6$. For the range $x<20$, the required value of $n$ is about 140 and the plot of this case cannot be shown in this figure.

In Figure 9, we show the accuracy of many selected previously mentioned approximations in terms of ARE and we leave the evaluation for interest readers.

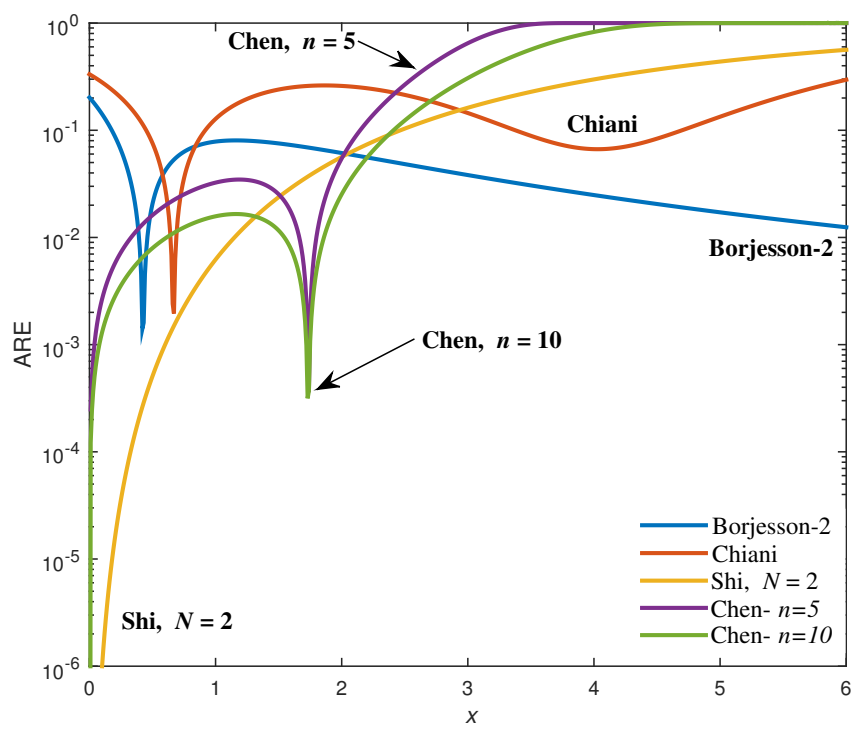

Figure 8. Comparison among Chiani, Benitez-MARE and Chen approximations with 8 and 12 terms.

\section{Tractability COMPARISON AND ApPlicATION Illustration}

In this section, three examples are provided to illustrate the mathematical tractability and applications of $Q$ function approximations. Note that we just limit our attention to $Q$ function approximations, which are applicable for algebraic manipulations related to communications performance derivation. Other $Q$ function approximations, which are efficient on programming, i.e., the Borjesson's approximations, will not be considered here. The first example is to derive the average SEP of differentially encoded QPSK over Nakagami- $m$ fading channels using the Benitez approximation. The second example is to show the usefulness of the PLBF approximation. And the last one is for performance derivation of emerging wireless networks.

\subsection{Average SEP of Differentially Encoded QPSK over Nakagami- $m$ Fading Channels}

This example is to give readers a particular illustration of how the general expression in (1) manipulated for the average SEP of DE-QPSK over single Nakagami$m$ fading. Recalling (2) and (3), we have

$$
\bar{P}_{\mathrm{s}}=4 I_{1}(m, \bar{\gamma})-8 I_{2}(m, \bar{\gamma})+I_{3}(m, \bar{\gamma})-4 I_{4}(m, \bar{\gamma}) .
$$

With the assumption that $Q_{\text {a-Benitez }}$ is used, $I_{n}(m, \bar{\gamma})$ can be approximated as follows:

$$
\begin{aligned}
I_{n}(m, \bar{\gamma})= & \int_{0}^{\infty} Q^{n}{ }_{\text {a-Benitez }}(\sqrt{\gamma}) f_{\gamma}(\gamma) d \gamma \\
= & e^{n c} \frac{m^{m}}{\bar{\gamma}^{m} \Gamma(m)} \\
& \times \int_{0}^{\infty} \gamma^{m-1} e^{-\left(n a+\frac{m}{\bar{\gamma}}\right) \gamma} e^{-n b \sqrt{\gamma}} \gamma^{m-1} d \gamma,
\end{aligned}
$$

where $a, b$, and $c$ are the absolute values of optimum fitting parameters in (36). 


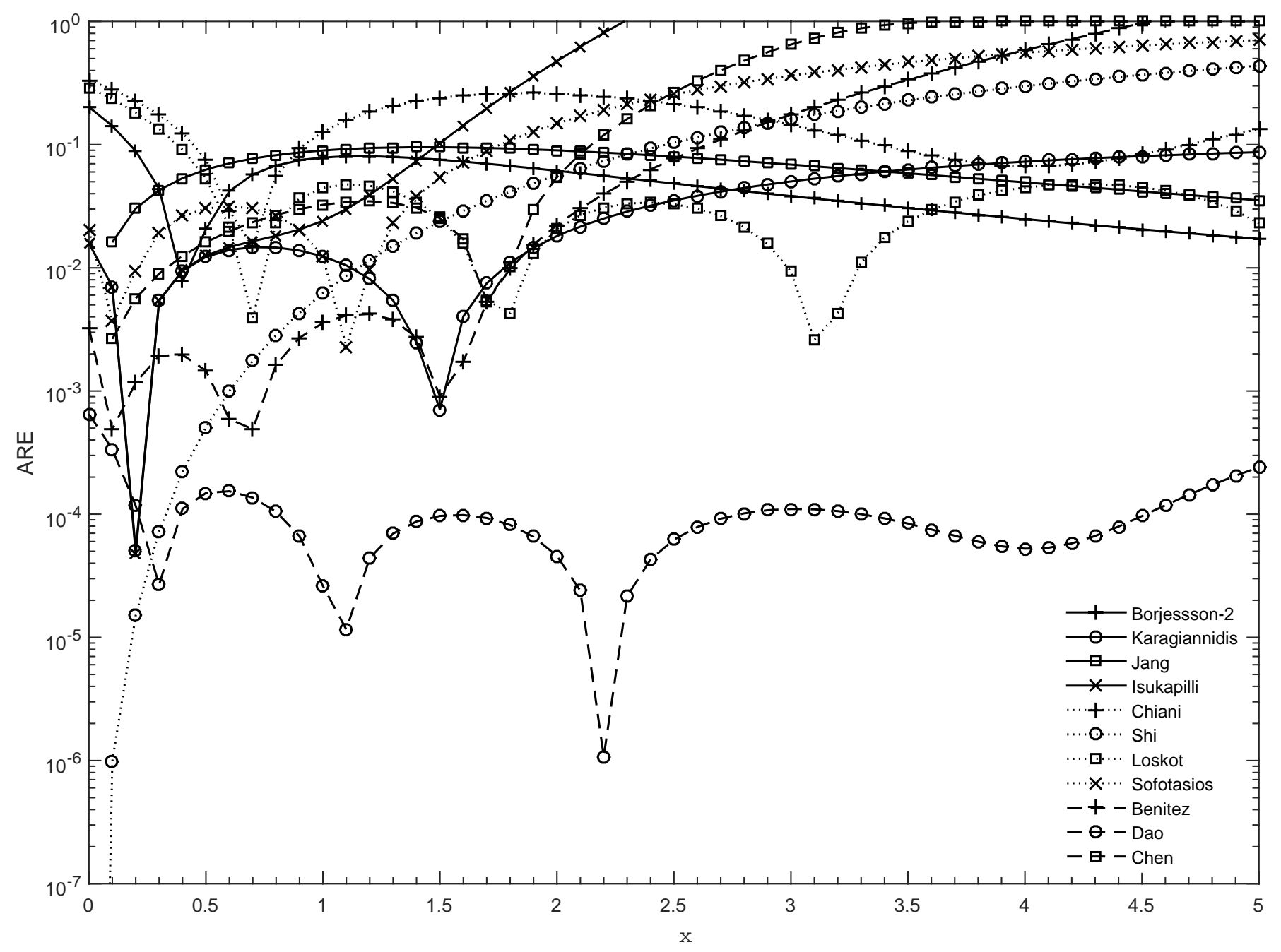

Figure 9. The accuracy of Q-function approximations in terms of ARE.

In $(41), f_{\gamma}(\gamma)$ denotes the PDF of Nakagami- $m$ fading channels given as $[1,2]$

$$
f_{\gamma}(\gamma)=\frac{m^{m} \gamma^{m-1}}{\bar{\gamma}^{m} \Gamma(m)} e^{-\frac{m}{\bar{\gamma}} \gamma}
$$

where $m \geq 0.5$ is the fading parameter; $\Gamma(\cdot)$ is the Gamma function [30]; $\bar{\gamma}$ is the average SNR per symbol, respectively.

By making a change of variable, i.e., $\gamma=x^{2},(41)$ can be rewritten as

$$
\begin{aligned}
I_{n}(m, \bar{\gamma})= & \frac{e^{n c}}{2} \frac{m^{m}}{\bar{\gamma}^{m} \Gamma(m)} \\
& \times \int_{0}^{\infty} x^{2(m-1)-1} e^{-\left(n a+\frac{m}{\bar{\gamma}}\right) x^{2}} e^{-n b x} d x \\
= & \frac{e^{n c}}{2} \frac{m^{m}}{\bar{\gamma}^{m} \Gamma(m)} J_{n}(m, \bar{\gamma}) .
\end{aligned}
$$

With the help of [33, eq. (3.462.1)], $J_{n}(m, \bar{\gamma})$ can be solved in closed-form as

$$
\begin{aligned}
J_{n}(m, \bar{\gamma})= & \exp \left(\frac{(n b)^{2}}{8 n a+\frac{8 m}{\bar{\gamma}}}\right)\left\{2 n a+\frac{2 m}{\bar{\gamma}}\right\}^{-(m-1)} \\
& \times \Gamma(2 m-2) D_{-2(m-1)}\left(\frac{n b}{\sqrt{2 n a+\frac{2 m}{\bar{\gamma}}}}\right),
\end{aligned}
$$

where $D_{v}($.$) denotes the parabolic cylinder func-$ tion [33, eq. (9.240)] which can be expressed in terms of confluent hypergeometric function ${ }_{1} F_{1}(., . ;$.$) [30,$ ch.13].

Combining (44), (43) and (40), we can obtain the closed-form expression of the average approximated SEP for DE-QPSK modulation over single Nakagami- $m$ fading channel. It is noted that the derivation approach here can be applied to many other $Q$ function approximation forms.

In Figure 10, we compare the simulated (exact) SEP with the approximated one for different $m$ ranging from 0.5 to 4.9 . It is clearly shown that the approximations are excellent agreement with the exact ones. 


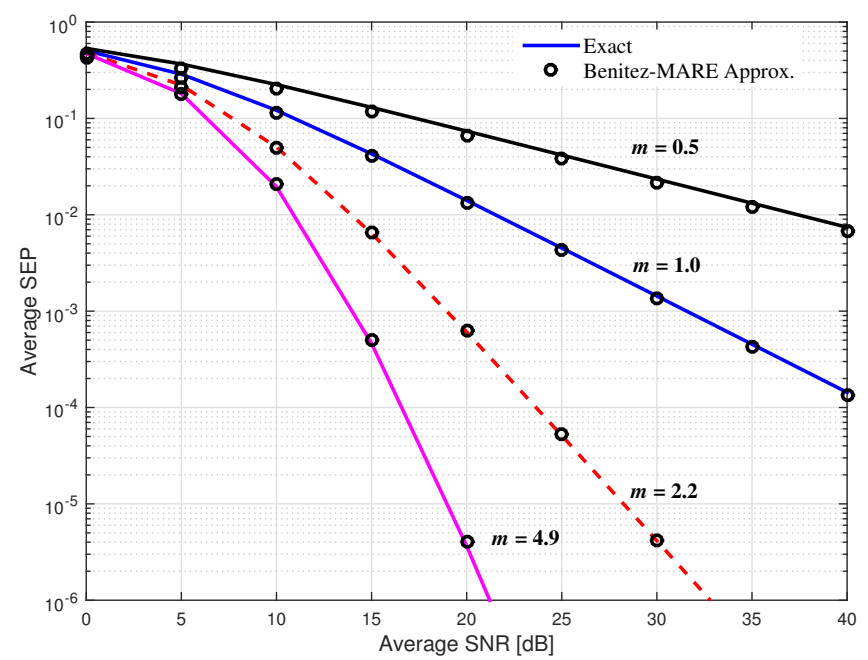

Figure 10. Average SEP for DE-QPSK over Nakagami- $m$ fading channel.

\subsection{Average BEP for Square M-QAM over log-normal Channels}

In this subsection, we will consider the average BEP for square M-QAM over log-normal channels given as [34]

$\overline{\mathrm{BER}}=\frac{2}{\sqrt{M} \log _{2} \sqrt{M}} \int_{0}^{\infty} \sum_{k=1}^{\log _{2} \sqrt{M}} \sum_{\ell=0}^{v_{k}} \phi_{\ell}^{k} \mathrm{Q}\left(\sqrt{\omega_{\ell} \gamma}\right) f_{\gamma}(\gamma) d \gamma$,

where $v_{k}, \phi_{\ell}^{k}$, and $\omega_{\ell}$ are defined respectively as

$$
\begin{gathered}
v_{k}=\left(1-2^{-k}\right) \sqrt{M}-1, \\
\phi_{\ell}^{k}=(-1)^{\left\lfloor\frac{\ell 2^{k-1}}{\sqrt{M}}\right\rfloor}\left(2^{k-1}-\left\lfloor\frac{\ell 2^{k-1}}{\sqrt{M}}+\frac{1}{2}\right\rfloor\right),
\end{gathered}
$$

and

$$
\omega_{\ell}=\frac{(2 \ell+1)^{2} 3 \log _{2} M}{M-1}
$$

We further define $\lfloor$.$\rfloor and f_{\gamma}(\gamma)$ as the floor function and the PDF of $\gamma$.

Interchanging the order of the integration and the summation, we have

$$
\overline{\mathrm{BER}}=\frac{2}{\sqrt{M} \log _{2} \sqrt{M}} \sum_{k=1}^{\log _{2} \sqrt{M}} \sum_{\ell=0}^{v_{k}} \underbrace{\int_{0}^{\infty} \phi_{\ell}^{k} \mathrm{Q}\left(\sqrt{\omega_{\ell} \gamma}\right) f_{\gamma}(\gamma) d \gamma}_{\mathcal{I}} .
$$

Observing the form of $f_{\gamma}(\gamma)$, we can see that it is very difficulty (or impossible) to employ MBRF or SEFF approximations to obtain the closed-form expression for (46) since exponential terms will make it more complicated. As a result, PLBF is a solution. In particular, substituting (39) into (45) and then taking the integral, we have

$$
\begin{aligned}
\mathcal{I}= & \phi_{\ell}^{k}-\phi_{\ell}^{k} \sum_{m=0}^{n} \sum_{p=0}^{n} \frac{(-1)^{m+p}\left(\begin{array}{c}
n \\
p
\end{array}\right)}{m !(n-m) !}\left(\frac{n}{12}\right)^{p / 2} \\
& \times\left(\frac{n}{2}-m\right)^{n-p} \omega_{\ell}^{\frac{p}{2}} \frac{10 / \ln (10)}{\sqrt{2 \pi \sigma^{2}}} \\
& \times \int_{0}^{\infty} \gamma^{\frac{p}{2}-1} \exp \left(-\frac{\left(10 \log _{10} \gamma-\mu\right)^{2}}{2 \sigma^{2}}\right) \\
& \times U\left[\sqrt{\omega_{\ell} \gamma}-\sqrt{\frac{12}{n}}\left(\frac{n}{2}-m\right)\right] d \gamma .
\end{aligned}
$$

Due to the step function, $U(x)$, we can rewrite $\mathcal{I}$ as follows:

$$
\begin{aligned}
& \mathcal{I}=\phi_{\ell}^{k}-\phi_{\ell}^{k} \sum_{m=0}^{n / 2} \sum_{p=0}^{n} \frac{(-1)^{m+p}\left(\begin{array}{c}
n \\
p
\end{array}\right)}{m !(n-m) !}\left(\frac{n}{12}\right)^{p / 2} \\
& \times\left(\frac{n}{2}-m\right)^{n-p} \beta_{M} \frac{p}{2} \frac{10 / \ln (10)}{\sqrt{2 \pi \sigma^{2}}} \\
& \times \underbrace{\int_{\gamma}^{\infty} \gamma^{\frac{p}{2}-1} \exp \left(-\frac{\left(10 \log _{10} \gamma-\mu\right)^{2}}{2 \sigma^{2}}\right)}_{\mathcal{I}_{1}} d \gamma \\
& -\phi_{\ell}^{k} \sum_{m=n / 2}^{n} \sum_{p=0}^{n} \frac{(-1)^{m+p}\left(\begin{array}{c}
n \\
p
\end{array}\right)}{m !(n-m) !}\left(\frac{n}{12}\right)^{p / 2} \\
& \times\left(\frac{n}{2}-m\right)^{n-p} \omega_{\ell}^{\frac{p}{2}} \frac{10 / \ln (10)}{\sqrt{2 \pi \sigma^{2}}} \\
& \times \underbrace{\int_{0}^{\infty} \gamma^{\frac{p}{2}-1} \exp \left(-\frac{\left(10 \log _{10} \gamma-\mu\right)^{2}}{2 \sigma^{2}}\right) d \gamma}_{\mathcal{I}_{2}},
\end{aligned}
$$

where $\gamma_{C}=\frac{12}{\omega_{\ell}}\left(\frac{n}{2}-m\right)^{2}$.

For $\mathcal{I}_{1}$, making use a change of variable, i.e., $t=$ $10 \log _{10} \gamma$, we have

$$
\begin{aligned}
\mathcal{I}_{1}= & \frac{\ln 10}{10} \sqrt{\frac{\pi \sigma^{2}}{2}} e^{\left(\frac{\ln 10}{20 \sqrt{2}}\right)^{2} p^{2} \sigma^{2}+\frac{\ln 10}{20} p \mu} \\
& \times\left[1+\operatorname{erf}\left(\frac{\ln 10}{20 \sqrt{2}} p \sigma+\frac{\mu-t_{C}}{\sqrt{2 \sigma^{2}}}\right)\right],
\end{aligned}
$$

where $t_{C}=10 \log _{10}\left[\frac{12}{\omega_{\ell} n}\left(\frac{n}{2}-m\right)^{2}\right]$.

$\mathcal{I}_{2}$ is of the form

$$
\mathcal{I}_{2}=\frac{\ln 10}{10} \sqrt{2 \pi \sigma^{2}} e^{\frac{\ln 10}{20} p \mu+\frac{\ln 10^{2}}{800} p^{2} \sigma^{2}} .
$$

Figure 11 studies the simulation (exact) BEP with the approximated BEP. It can be seen that the approximate $\mathrm{BEP}$ is very close to the exact SER for both 4-QAM and 16-QAM. We can see that the accuracy of the BEP approximation improves as $n$ increases. For example, the case of $n=9$ outperforms the other two cases, $n=6$ and $n=3$ in all range of SNRs. Figure 11 also indicates that the BEP approximations does not work well for large SNRs with small $n$. 


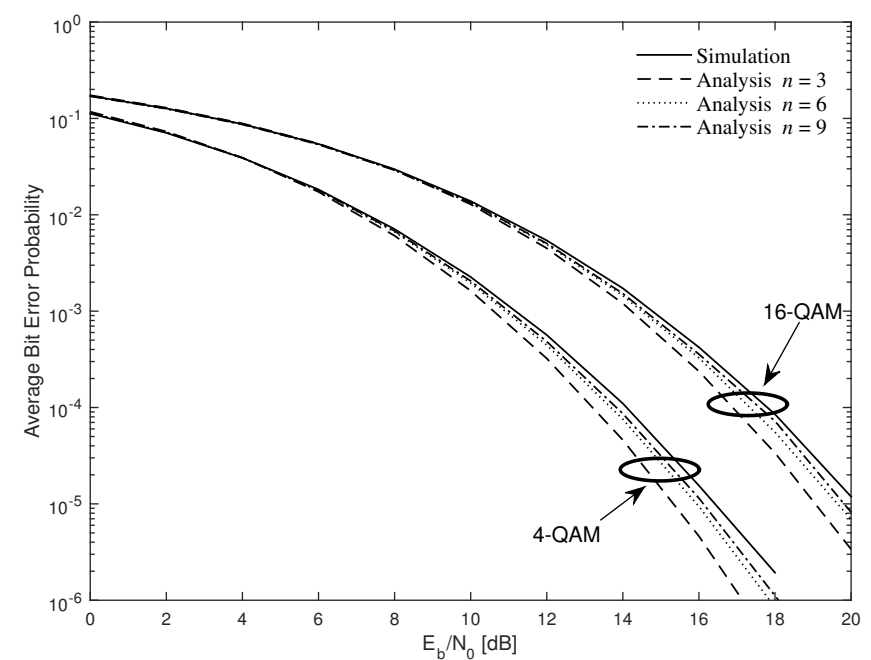

Figure 11. Average BEP for square $M$-QAM over log-normal fading channel, $\sigma=3$.

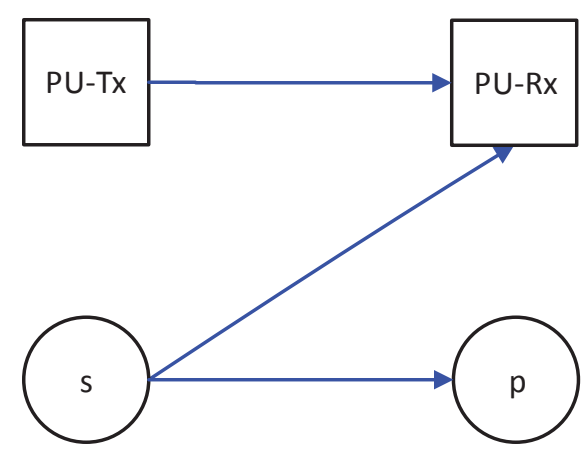

Figure 12. System model: the cognitive underlay network.

\subsection{Average BEP of cognitive underlay networks}

Recently, cognitive radio is considered as a promising technique to improve the spectrum utilization due to the ineffective of the fixed spectrum allocation policy by allowing cognitive (un-licensed) networks operating on the same spectrum with licensed networks [35]. The underlay approach is an efficient cognitive approach as compared with the overlay and interweave approach since it is able to provide concurrent transmission for un-licensed networks [36-38]. Here, we consider the underlay cognitive networks as shown in Figure 12.

To protect the primary network's communication, the transmit power of the secondary network, $P$, is a function of the channel gain of the interference link from the secondary source (s) to the primary receiver (p). Let us denote $h$ and $f$ as the channel coefficients of the channels from $\mathrm{s} \rightarrow \mathrm{d}$ and from $\mathrm{s} \rightarrow \mathrm{p}$, respectively, we have [39]

$$
P=\min \left(\frac{I_{\mathrm{p}}}{|f|^{2}}, P_{m}\right),
$$

where $I_{\mathrm{p}}$ denotes the interference tolerable interference power at the primary receiver and $P_{m}$ is the maximum transmission power, leading to the instantaneous SNR as follows [40]:

$$
\gamma=\min \left(\frac{I_{\mathrm{p}}}{N_{0}} \frac{|h|^{2}}{|f|^{2}}, \frac{P_{m}}{N_{0}}|h|^{2}\right) .
$$

Over Rayleigh fading channel, the CDF and PDF of $\gamma$ are given respectively as follows [39]:

$$
F_{\gamma}(\gamma)=1-e^{-\frac{\gamma}{\alpha_{1}}}+\alpha_{2} \frac{\gamma}{\gamma+\alpha_{3}} e^{-\frac{\gamma}{\alpha_{1}}}
$$

and

$$
\begin{aligned}
f_{\gamma}(\gamma)= & \frac{1}{\alpha_{1}} e^{-\frac{\gamma}{\alpha_{1}}}+\frac{\alpha_{2}}{\gamma+\alpha_{3}} e^{-\frac{\gamma}{\alpha_{1}}} \\
& -\frac{\alpha_{2}}{\alpha_{1}} \frac{\gamma}{\gamma+\alpha_{3}} e^{-\frac{\gamma}{\alpha_{1}}}-\frac{\alpha_{2} \gamma}{\left(\gamma+\alpha_{3}\right)^{2}} e^{-\frac{\gamma}{\alpha_{1}}},
\end{aligned}
$$

where

$$
\begin{aligned}
\alpha_{1} & =\frac{P_{m}}{N_{0}} \mathbb{E}\left\{|h|^{2}\right\} \\
\alpha_{2} & =e^{\frac{I_{p} / N_{0}}{P_{m}} \mathbb{E}\left\{|f|^{2}\right\}} \\
\alpha_{3} & =\frac{I_{\mathrm{p}}}{N_{0}} \frac{\mathbb{E}\left\{|h|^{2}\right\}}{\mathbb{E}\left\{|f|^{2}\right\}} .
\end{aligned}
$$

We now can write the average bit error probability for secondary networks as follows:

$$
\mathrm{BER}=\int_{0}^{\infty} a Q(\sqrt{b \gamma}) f_{\gamma}(\gamma) d \gamma,
$$

where $a$ and $b$ are modulation-dependent parameters [41]. For example, $a=1$ and $b=2$ for BPSK. For other modulation schemes, the combination of $a$ and $b$ can be given in [41, Table 6.1].

It is noted that (56) does not exist closed-form expression with the exact form of $Q(\cdot)$. To deal with this problem, approximations of $Q(\cdot)$ should be used. However, except Chiani approximations, i.e, (27), other approximations mentioned above do not make (56) integrable. In particular, plugging (27) into (56), we have following equation:

$$
\begin{aligned}
\mathrm{BER}= & \int_{0}^{\infty}\left(\frac{1}{12} e^{-\gamma}+\frac{1}{4} e^{-\frac{2 \gamma}{3}}\right) \\
& \times\left[\frac{1}{\alpha_{1}} e^{-\frac{\gamma}{\alpha_{1}}}+\frac{\alpha_{2}}{\gamma+\alpha_{3}} e^{-\frac{\gamma}{\alpha_{1}}}\right. \\
& \left.-\frac{\alpha_{2}}{\alpha_{1}} \frac{\gamma}{\gamma+\alpha_{3}} e^{-\frac{\gamma}{\alpha_{1}}}-\frac{\alpha_{2} \gamma}{\left(\gamma+\alpha_{3}\right)^{2}} e^{-\frac{\gamma}{\alpha_{1}}}\right] d \gamma .
\end{aligned}
$$

After rearrangement and grouping like terms, we have

$$
\begin{aligned}
\text { BER }= & \frac{1}{12 \alpha_{1}} \mathcal{I}_{1}\left(1+\frac{1}{\alpha_{1}}\right)+\frac{1}{4 \alpha_{1}} \mathcal{I}_{1}\left(\frac{4}{3}+\frac{1}{\alpha_{1}}\right) \\
& +\frac{\alpha_{2}}{12} \mathcal{I}_{2}\left(\alpha_{3}, 1+\frac{1}{\alpha_{1}}\right)+\frac{\alpha_{2}}{4} \mathcal{I}_{2}\left(\alpha_{3}, \frac{4}{3}+\frac{1}{\alpha_{1}}\right) \\
& -\frac{\alpha_{2}}{12 \alpha_{1}} \mathcal{I}_{3}\left(\alpha_{3}, 1+\frac{1}{\alpha_{1}}\right)-\frac{\alpha_{2}}{4 \alpha_{1}} \mathcal{I}_{3}\left(\alpha_{3}, \frac{4}{3}+\frac{1}{\alpha_{1}}\right) \\
& -\frac{\alpha_{2}}{12} \mathcal{I}_{4}\left(\alpha_{3}, 1+\frac{1}{\alpha_{1}}\right)-\frac{\alpha_{2}}{4} \mathcal{I}_{4}\left(\alpha_{3}, \frac{4}{3}+\frac{1}{\alpha_{1}}\right) .
\end{aligned}
$$

In (58), with the help of [33, eq. (3.353.2)], [33, eq. (3.353.3)] and [33, eq. (3.353.5)], $\mathcal{I}_{k}$ with $k=1,2,3,4$ 


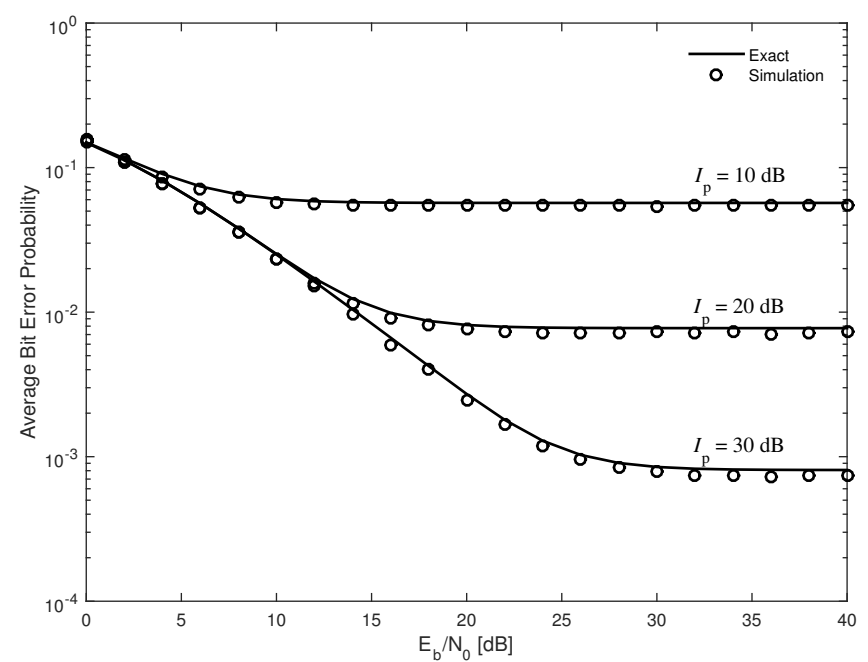

Figure 13. Average BEP for BPSK of underlay cognitive networks, $\mathbb{E}\left\{|h|^{2}\right\}=1$ and $\mathbb{E}\left\{|f|^{2}\right\}=3$.

is calculated respectively as follows:

$$
\begin{aligned}
\mathcal{I}_{1}\left(\phi_{2}\right) & =\int_{0}^{\infty} e^{-\gamma \phi_{2}} d \gamma=\frac{1}{\phi_{2}}, \\
\mathcal{I}_{2}\left(\phi_{1}, \phi_{2}\right) & =\int_{0}^{\infty} \frac{e^{-\phi_{2} \gamma}}{\gamma+\phi_{1}} d \gamma \\
& =e^{\phi_{1} \phi_{2}} \Gamma\left(0, \phi_{1} \phi_{2}\right), \\
\mathcal{I}_{3}\left(\phi_{1}, \phi_{2}\right) & =\int_{0}^{\infty} \frac{\gamma}{\gamma+\phi_{1}} e^{-\phi_{2} \gamma} d \gamma \\
& =\frac{1}{\phi_{1}}-\phi_{2} e^{-\phi_{1} \phi_{2}} \Gamma\left(0, \phi_{1} \phi_{2}\right), \\
\mathcal{I}_{4}\left(\phi_{1}, \phi_{2}\right) & =\int_{0}^{\infty} \frac{\gamma}{\left(\gamma+\phi_{1}\right)^{2}} e^{-\phi_{2} \gamma} d \gamma \\
& =\left(1+\phi_{1} \phi_{2}\right) \mathrm{e}^{\phi_{1} \phi_{2}} \Gamma\left(0, \phi_{1} \phi_{2}\right)-1 .
\end{aligned}
$$

In Figure 13, we compare the simulation BEP and the approximated BEP using Chiani approximations for three cases of $I_{p}$, i.e., $I_{p}=10 \mathrm{~dB}, I_{p}=20 \mathrm{~dB}$, and $I_{p}=$ $30 \mathrm{~dB}$ over the range of $0-40 \mathrm{~dB}$ of operating SNRs. It can be seen that the approximated results are in good agreement with the simulated results in all range of SNRs. The gap between the the exact result and the approximated is very small and does not depend on neither $I_{\mathrm{p}}$ nor $E_{b} / N_{0}$.

\section{Computational Complexity}

To evaluate the complexity of all approximations, we use an in-direct approach by measuring the average time to compute approximations. For the same argument of $x$, i.e., an equidistant vector from 1 to 10 with 10000 elements, Table III and Figure 14 provide the average time in computing for all approximations. We can see that in terms of average time, MRBF outperforms SEFF, which, in turns, outperforms PNBF.
Table III

Average Time for All Approximations

\begin{tabular}{|l|c|}
\hline Approximations & Average time [s] \\
\hline$Q_{\text {a-Borjessson }}(x)$ & 0.0606 \\
\hline$Q_{\text {a-Karagiannidis }}(x)$ & 0.1496 \\
\hline$Q_{\text {a-Jang }}(x)$ & 0.2429 \\
\hline$Q_{\text {a-Isukapilli }}(x)$ & 0.5745 \\
\hline$Q_{\text {a-Chiani }}(x)$ & 0.8872 \\
\hline$Q_{\text {a-Shi }}(x)$ & 1.0161 \\
\hline$Q_{\text {a-Loskot }}(x)$ & 1.1403 \\
\hline$Q_{\text {a-Sofotasios }}(x)$ & 1.2318 \\
\hline$Q_{\text {a-Benitez }}(x)$ & 1.3050 \\
\hline$Q_{\text {a-Dao }}(x)$ & 1.7795 \\
\hline$Q_{\text {a-Chen }}(x)$ & 5.0110 \\
\hline
\end{tabular}

\section{Conclusion}

In this paper, we have presented an overview about the approximation of the one-dimensional Gaussian $Q$-function. In spite of many different approaches used by researchers, there are just three approximation forms, namely The Mills'-ratio-based form, the sum-ofexponential-function-based form and the polynomialbased form. Via some illustrating examples, we also discussed their accuracy and tractability. The obtained results are systematically presented either by graphs or by tables, where necessary information about the performance of each method is shown. These illustrating examples have been chosen by their representativeness and their complexity in order to show that by using accurate approximations of $Q$-function, the performance analysis of digital communication systems becomes much simpler. We can therefore conclude that these approximations can help simplify the design procedure of digital communication systems.

\section{REFERENCES}

[1] J. G. Proakis and M. Salehi, Digital Communications, 5th ed. New York: McGraw-Hill, 2008.

[2] M. K. Simon and M. S. Alouini, Digital communication over fading channels, 2nd ed., ser. Wiley series in telecommunications and signal processing. Hoboken, N.J.: John Wiley and Sons, 2005.

[3] M. K. Simon, "A simpler form of the Craig representation for the two-dimensional joint Gaussian $Q$-function," IEEE Communications Letters, vol. 6, no. 2, pp. 49-51, Feb. 2002.

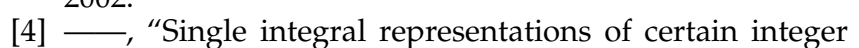
powers of the Gaussian $Q$-function and their application," IEEE Communications Letters, vol. 6, no. 12, pp. 532-534, Dec. 2002.

[5] R. Li and P. Y. Kam, "Averages of the product of two gaussian $q$-functions over fading statistics and applications," IEEE Communications Letters, vol. 11, no. 1, pp. 58-60, Jan. 2007.

[6] J. W. Craig, "A new, simple and exact result for calculating the probability of error for two-dimensional signal constellations," in Proc. of the IEEE 1991 Military 


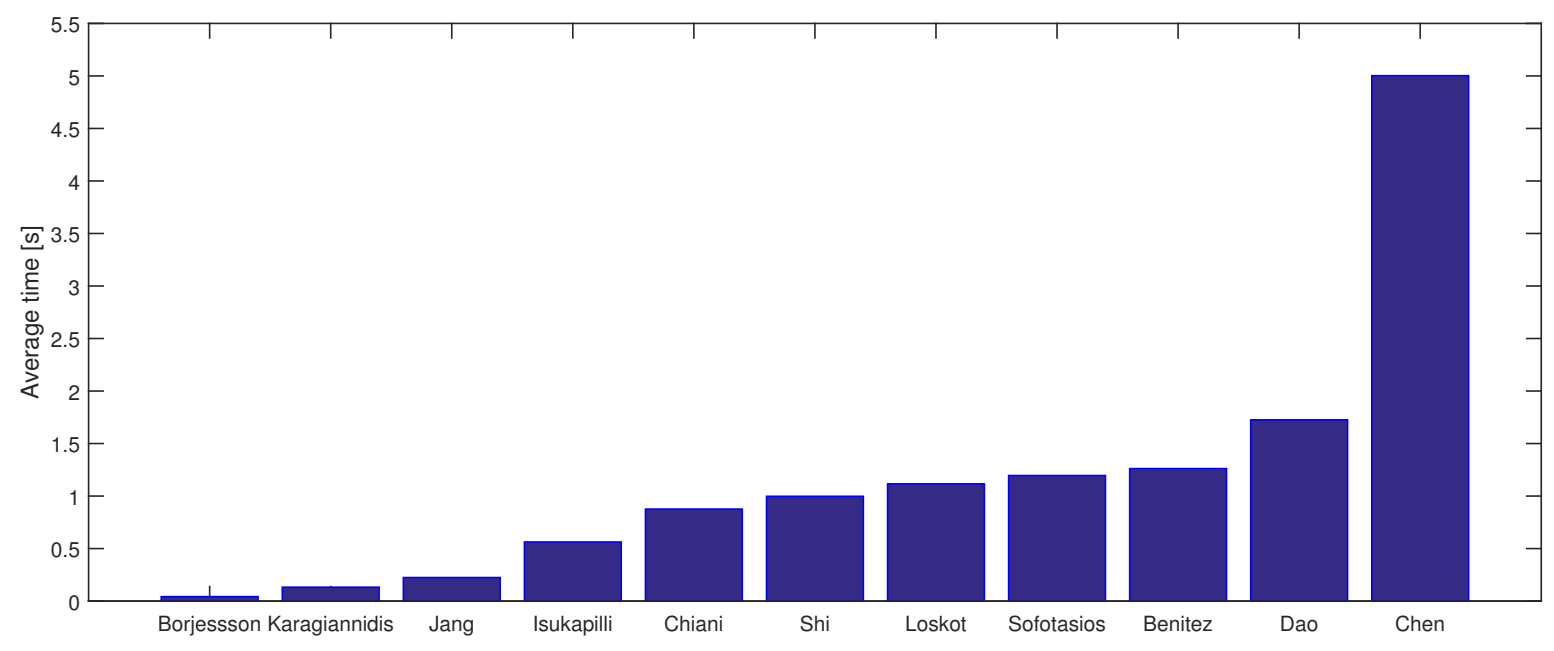

Figure 14. Average time for all $Q$ function approximations.

Communications Conference (MILCOM '91): Military Communications in a Changing World, vol. 2, 1991, pp. 571-575.

[7] R. K. Mallik, "Average of product of two Gaussian $q$ functions and its application to performance analysis in Nakagami fading," IEEE Transactions on Communications, vol. 56, no. 8, pp. 1289-1299, Aug. 2008.

[8] N. C. Beaulieu, "A useful integral for wireless communication theory and its application to rectangular signaling constellation error rates," IEEE Transactions on Communications, vol. 54, no. 5, pp. 802-805, May 2006.

[9] R. M. Radaydeh and M. M. Matalgah, "Results for infinite integrals involving higher-order powers of the Gaussian $Q$-function with application to average SEP analysis of DE-QPSK," IEEE Communications Letters, vol. 7, no. 3, pp. 793-798, Mar. 2008.

[10] Y. Chen and N. C. Beaulieu, "Solutions to infinite integrals of Gaussian $Q$-function products and some applications," IEEE Communications Letters, vol. 11, no. 11, pp. 853-855, Nov. 2007.

[11] P. Borjesson and C.-E. Sundberg, "Simple approximations of the error function $Q(x)$ for communications applications," IEEE Transactions on Communications, vol. 27, no. 3, pp. 639-643, Mar. 1979.

[12] G. K. Karagiannidis and A. S. Lioumpas, "An improved approximation for the Gaussian $Q$-function," IEEE Communications Letters, vol. 11, no. 8, pp. 644-646, Aug. 2007.

[13] M. Chiani, D. Dardari, and M. K. Simon, "New exponential bounds and approximations for the computation of error probability in fading channels," IEEE Transactions on Wireless Communications, vol. 2, no. 4, pp. 840-845, Jul. 2003.

[14] Y. Isukapalli and B. D. Rao, "An analytically tractable approximation for the Gaussian $Q$-function," IEEE Communications Letters, vol. 12, no. 9, Sep. 2008.

[15] P. Loskot and N. C. Beaulieu, "Further results on prony approximation for evaluation of the average probability of error," in Proc. of the IEEE International Conference on Communications (ICC '08), 2008.

[16] Y. Chen and N. C. Beaulieu, "A simple polynomial approximation to the Gaussian $Q$-function and its application," IEEE Communications Letters, vol. 13, no. 2, pp. 124-126, Feb. 2009.

[17] P. Loskot and N. C. Beaulieu, "Prony and polynomial approximations for evaluation of the average probability of error over slow-fading channels," IEEE Transactions on Vehicular Technology, vol. 58, no. 3, pp. 1269-1280, Mar. 2009.

[18] P. C. Sofotasios and S. Freear, "Novel expressions for the Marcum and one dimensional $Q$-functions," in Proc. of the IEEE International Conference on Wireless Information
Technology and Systems (ICWITS'10), 2010, pp. 736-740.

[19] W. M. Jang, "A simple upper bound of the Gaussian $Q$-function with closed-form error bound," IEEE Communications Letters, vol. 14, no. 2, p. 157, Feb. 2011.

[20] L. M. Benitez and F. Casadevall, "Versatile, accurate, and analytically tractable approximation for the gaussian $Q$ function," IEEE Transactions on Communications, vol. 59, no. 4, pp. 917-922, Apr. 2011.

[21] Q. Shi and Y. Karasawa, "An accurate and efficient approximation to the Gaussian $Q$-function and its applications in performance analysis in Nakagami- $m$ fading," IEEE Communications Letters, no. 49, pp. 1-3, May 2011.

[22] M. K. Simon, Probability distributions involving Gaussian random variables: a handbook for engineers and scientists. Boston: Kluwer Academic Publishers, 2002.

[23] - "A new twist on the Marcum $Q$-function and its application," IEEE Communications Letters, vol. 2, no. 2, pp. 39-41, Feb. 1998.

[24] A. H. Nuttall, "Some integrals involving the $Q_{m}$ function," IEEE Transactions on Information Theory, pp. 95-96, Jan. 1975.

[25] D. S. Mitrinovic and P. M. Vasic, Analytic inequalities. Springer, 1970, vol. 61.

[26] A. Baricz, "Mills's ratio: Monotonicity patterns and functional inequalities," Journal of Mathematical Analysis and Applications, pp. 1362-1370, Apr. 2008.

[27] G. T. F. d. Abreu, "Jensen-cotes upper and lower bounds on the Gaussian $Q$-function and related functions," IEEE Transactions on Communications, vol. 57, no. 11, pp. 33283338, Nov. 2009.

[28] J. S. Dyer and S. A. Dyer, "Corrections to, and comments on, "an improved approximation for the Gaussian $Q$ function"," IEEE Communications Letters, vol. 12, no. 4, p. 231, Apr. 2008.

[29] N. M. Steen, G. D. Byrne, and E. M. Gelbard, "Gaussian quadratures for the integrals," Mathematics of Computation, vol. 23, no. 107, pp. 661-671, 1969.

[30] M. Abramowitz, I. A. Stegun, and K. (Firm), Handbook of mathematical functions with formulas, graphs, and mathematical tables, 10th ed. Washington, D.C.: U.S. Dept. of Commerce : U.S. G.P.O., 1972.

[31] P. Dao Ngoc, U. Nguyen Quang, H. Nguyen Xuan, and R. McKay, "Evolving approximations for the gaussian Qfunction by genetic programming with semantic based crossover," in Proc. of the 2012 IEEE Congress onEvolutionary Computation (CEC), 2012, pp. 1-6.

[32] A. Papoulis and S. U. Pillai, Probability, random variables, and stochastic processes, 4th ed. Boston: McGraw-Hill, 2002.

[33] I. S. Gradshteyn, I. M. Ryzhik, A. Jeffrey, and D. Zwill- 
inger, Table of integrals, series and products, 7th ed. Amsterdam ; Boston: Elsevier, 2007.

[34] K. Cho and D. Yoon, "On the general BER expression of one- and two-dimensional amplitude modulations," IEEE Transactions on Communications, vol. 50, no. 7, pp. 1074-1080, Jul. 2002.

[35] A. Goldsmith, S. A. Jafar, I. Maric, and S. Srinivasa, "Breaking spectrum gridlock with cognitive radios: An information theoretic perspective," Proceedings of the IEEE, vol. 97, no. 5, pp. 894-914, May 2009.

[36] T. Q. Duong, V. N. Q. Bao, and H.-J. Zepernick, “Exact outage probability of cognitive AF relaying with underlay spectrum sharing," Electronics Letters, pp. 1001-1002, Aug. 2011.

[37] V. N. Q. Bao and D. H. Bac, "A unified framework for performance analysis of DF cognitive relay networks under interference constraints," in 2011 International Conference on ICT Convergence (ICTC). IEEE, 2011, pp. 537 $-542$.

[38] V. N. Q. Bao, T. T. Thanh, N. T. Duc, and V. D. Thanh, "Spectrum sharing-based multihop decode-and-forward relay networks under interference constraints: Performance analysis and relay position optimization," Journal of Communications and Networks, vol. 15, no. 3, pp. 266275, Jul. 2013.

[39] V. N. Q. Bao and T. Q. Duong, "Outage analysis of cognitive multihop networks under interference constraints," IEICE Transactions on Communications, vol. E95-B, no. 03, pp. 1019-1022, Mar. 2012.

[40] T. Q. Duong, Y. Phee Lep, V. N. Q. Bao, M. Elkashlan, and Y. Nan, "Cognitive relay networks with multiple primary transceivers under spectrum-sharing," IEEE Signal Processing Letters, vol. 19, no. 11, pp. 741-744, Sep. 2012.

[41] A. Goldsmith, Wireless communications. Cambridge University Press, 2005.

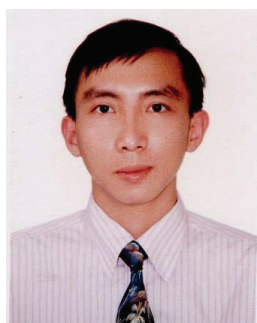

Vo Nguyen Quoc Bao was born in Nha Trang, Khanh Hoa Province, Vietnam. He received the B.E. and M.Eng. degree in electrical engineering from Ho Chi Minh City University of Technology (HCMUT), Vietnam, in 2002 and 2005, respectively, and Ph.D. degree in electrical engineering from University of Ulsan, South Korea, in 2009. In 2002, he joined the Department of Electrical Engineering, Posts and Telecommunications Institute of Technology (PTIT), as a lecturer. Since February 2010, he has been with the Department of Telecommunications, PTIT, where he is currently an Associate Professor. His major research interests are modulation and coding techniques, MIMO systems, combining techniques, cooperative communications, cognitive radio, energy harvesting. Dr. Bao is a member of Korea Information and Communications Society (KICS), The Institute of Electronics, Information and Communication Engineers (IEICE) and The Institute of Electrical and Electronics Engineers (IEEE). He is currently serving as the Editor of Transactions on Emerging Telecommunications Technologies (Wiley ETT). He is also a Guest Editor of EURASIP Journal on Wireless Communications and Networking, special issue on "Cooperative Cognitive Networks" and IET Communications, special issue on "Secure Physical Layer Communications".

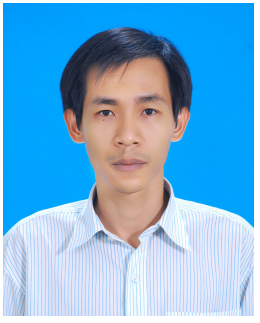

Luu Pham Tuyen received the B.Eng. degree in Telecommunications Engineering from Posts and Telecommunications Institute of Technology in 2003. From 2009, he has been working toward the M.Eng. degree at Posts and Telecommunications Institute of Technology (PTIT). His research interests include the areas of mobile communication networks and performance analysis.

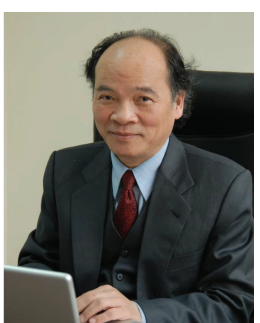

Huu-Tue Huynh was born in Hue, Viet Nam. He received the Sc.D. degree in 1972 from Laval University, Canada, where from 1969 to 2004 he was a faculty member of the Department of Electrical and Computer Engineering. In 2004, he left Laval University to become Chairman of the Department of Data Processing at The College of Technology of the Vietnam National University, Hanoi. During the period 2007-2011, he was the President of Bac Ha International University, Vietnam. He is now a research professor at the School of Electrical Engineering, International University, Vietnam National University, Ho Chi Minh City, Vietnam. He was an Invited Guest at The AT\&T Information Systems in Neptune, N.J. in 1984 and has been invited to give lectures at several Universities in Europe, America as well as in Asia. Professor Huynh is author and coauthor of two books and more than two hundred papers in Information Processing. He has served as Consultant to a number of Canadian Government Agencies and Industries. His research interests cover stochastic simulation techniques, information processing, fast algorithms and architectures with applications to finance and to communications 